\title{
Gute deutsche Politik
}

Was musste sich Wolfgang Schäuble seit der erfolgreichen Erpressung von Alexis Tsipras und dessen Regierung nicht alles für Kritik anhören: Er habe die Demokratie in Europa beschädigt, er habe dem Ansehen Deutschlands im Ausland geschadet und ja, vor allem die „europäische Idee“ habe er beschädigt. Der Mann scheint nur noch unterwegs zu sein, um alles Schöne und Edle zu beschädigen. Konsequenterweise forderte eine Onlinepetition seinen Rücktritt. Was aber, wenn Schäuble nicht einfach nur starrsinnig, gemein und unsozial ist? Was, wenn er einfach nur gute deutsche Politik macht?

Es hat sich einiges geändert in der deutschen Politik. Gegenüber Griechenland ist das nur besonders deutlich geworden. Seit Jahrzehnten profitiert die deutsche Wirtschaft mehr von der EU (und früher von der EWG) als die Wirtschaft jedes anderen Landes. Und der deutsche Staat profitiert über seine Steuereinnahmen ordentlich mit. Egal welche Partei den Kanzler stellte, die deutsche Europapolitik setzte auf Konsens. Klaglos wurde die Rolle des größten Nettozahlers der Gemeinschaft akzeptiert. Was an die Union gezahlt wurde, war nicht der Rede wert im Vergleich mit dem, was durch die Union verdient wurde. Gegenüber der Syriza-Regierung wurde mit dieser Konsenspolitik demonstrativ gebrochen. Obwohl es bei einer ganzen Reihe von Mitgliedsländern erhebliche Bedenken gab, erzwangen Merkel und Schäuble eine kompromisslose Haltung der Eurozone: Mit weniger als einer bedingungslosen
Kapitulation der griechischen Regierung gaben sie sich nicht zufrieden. Deutschland hat mit der Konsenspolitik nicht nur gebrochen. Dieser Bruch sollte auch für alle gut sichtbar sein - als Warnung.

Demokratie ist schön und gut. Ohne freie Wahlen kann man nicht EU-Mitglied werden. Nur darf daraus nicht abgeleitet werden, dass eine frei gewählte Regierung, die einen verschuldeten Staat übernimmt, auch die Politik machen kann, die sie für richtig hält. Merkel und Schäuble forderten "Reformen“, also noch mehr von genau dem Verarmungsprogramm, das in den letzten fünf Jahren zu einer knapp 30-prozentigen Schrumpfung des griechischen Sozialprodukts geführt hat. Mario Draghi als EZBChef sorgte dafür, dass den griechischen Banken die Refinanzierungsmöglichkeiten genommen wurden. Ohne die geht aber jede Bank in kürzester Zeit Bankrott. Die griechische Regierung hatte nur noch die Wahl entweder ganz schnell eine Parallelwährung auszugeben, was zu einem chaotischen Defacto-Ausstieg aus dem Euro geführt hätte, oder aber alles zu unterschreiben, was man ihr vorgelegt hat. Schäuble hat nicht die Demokratie in Europa beschädigt (wann und wo existierte diese fantastische unbeschädigte Demokratie eigentlich?), er hat lediglich deutlich gemacht, wie im Europa des Euro und der deutschen Dominanz die Demokratie funktioniert.

Aber wurde nicht genau damit die „europäische Idee" beschädigt? Dass EWG und EU nach dem Zweiten Weltkrieg den westlichen Teil Europas vom Nationalismus 
befreit und endlich Frieden und Völkerverständigung gebracht hätten, wird zwar immer wieder in die Welt hinausposaunt. Auch von vielen Linken wird es geglaubt, die deshalb vor jeder grundsätzlichen Kritik an der EU zurückschrecken, da sie nicht dem Nationalismus in die Hände spielen wollen. Ein kurzer Blick in die Geschichte zeigt aber ein anderes Bild. Für den Frieden in Europa sorgte einerseits der Kalte Krieg für innerwestliche Konflikte etwa zwischen Deutschland und Frankreich war kein Platz mehr - und andererseits die atomare Bedrohung: Ein Krieg zwischen den Blöcken hätte zum atomaren Inferno geführt. Der Friede in Europa war nicht die Folge, sondern die Voraussetzung für die Gründung der EWG im Jahre 1957.

Diese Gründung hatte eine politische und eine ökonomische Dimension. Politisch war die Bundesrepublik - 12 Jahre nach der Niederlage des deutschen Faschismus und ohne irgendwelche Reparationen für den Angriffskrieg und die Vielzahl der dabei begangenen Verbrechen leisten zu müssen - als EWG-Mitglied endlich wieder ein vollwertiger Teil der „westlichen Welt“. Für den Westen war dies die gelungene Einbindung der Bundesrepublik, die nur wenige Jahre vorher von Stalin das Angebot Wiedervereinigung gegen Neutralität erhalten hatte. Ökonomisch: Nachdem der Bundesrepublik in der Londoner Schuldenkonferenz 1953 bereits ein großer Teil ihrer Schulden erlassen und die Rückzahlung des Restes auf zum Teil 50 Jahre gestreckt worden war, verschaffte die EWG der schnell wachsenden deutschen Industrie den größeren Markt, den sie unbedingt brauchte. Frankreich und Italien versprachen sich von der EWG eine nachholende Entwicklung ihrer Wirtschaft und insbesondere Frankreich auch Unterstützung für seinen großen und maroden Agrarsektor.

Von der EWG profitierten in einem günstigen weltwirtschaftlichen Umfeld alle - aber der bei Weitem größte Profiteur war die Bundesrepublik. Dies galt auch noch für die Einführung des Euro, nur dass die Abstände zunahmen. Während der Süden nur wenig profitierte, räumte der einheitliche Währungsraum für das deutsche Kapital nicht nur die innereuropäischen Hindernisse beiseite, auch jenseits der EU verschaffte der Euro, der tendenziell schwächer als die D-Mark ist, der deutschen Exportwalze nicht zu unterschätzende Konkurrenzvorteile und längerfristig kann er sogar den Dollar als alleiniges Weltgeld angreifen. Allerdings waren die deutschen Regierungen nicht mehr bereit, die enormen Vorteile, die aus dem Euro resultierten, mit den schwächeren Ländern zu teilen. Sollten die sich doch mit der für sie viel zu starken Währung alleine herumschlagen, irgendeine Art von „Transferunion“ kam für Deutschland nicht infrage. Der Euro leidet nicht an einem oft beschworenen Konstruktionsfehler, den blinde Ingenieure irgendwie übersehen haben und jetzt nicht zugeben wollen. Die Konstruktion war so gewollt, nicht nur von der deutschen Regierung, sondern auch von anderen Regierungen, die ihre Länder für stark hielten und im Euro auch ein willkommenes Disziplinierungsinstrument auch nach innen sahen. Die Schwächeren stimmten zu, da sie hofften, dass alles schon nicht so schlimm werden würde, aber vor allem, weil die Alternative draußen zu bleiben, als noch viel unangenehmer erschien.

Diese neue, nur auf den Vorteil der starken Länder zielende Politik wurde in aller Brutalität gegenüber Griechenland durchexerziert. Dabei ging es gar nicht in erster Linie darum, Griechenland bis aufs letzte auszuquetschen. Auch wenn ein Sahnehäubchen, wie die Übernahme griechischer Flughäfen durch die Frankfurter Flughafengesellschaft Fraport zum Schnäppchenpreis gerne mitgenommen und politisch unterstützt wird 
- im Memorandum, das die griechische Regierung im August 2015 unterschreiben musste, wurde die Flughafenprivatisierung als besonders dringlicher Punkt explizit aufgeführt. Auch Merkel und Schäuble ist klar, dass die kleine, durch immer neue Sparorgien geschwächte griechische Ökonomie, niemals in der Lage sein wird, die enormen Schulden substanziell abzubauen. Entweder es kommt irgendwann ein Schuldenschnitt (oder eine enorme Laufzeitverlängerung bei gleichzeitig minimalen Zinsen, was faktisch auf das Gleiche hinausläuft) oder die sogenannten Rettungsprogramme - neue höhere Kredite, um die alten mitsamt Zinsen zu tilgen - werden zur Dauereinrichtung. Dieses Offensichtliche, was bis in die Talkshows vorgedrungen ist, hat auf Seite der "Institutionen" bis jetzt nur IWF-Chefin Lagarde ausgesprochen.

Entgegen der landläufigen Meinung hat der deutsche Staat bis jetzt fast nichts für Griechenland gezahlt. Die verschiedenen „Hilfspakete“ sind Kredite, für die Griechenland Zinsen zahlen muss. "Gerettet" wurden die privaten Banken, die ursprünglich Kredite an den griechischen Staat vergeben hatten. Diese Kredite wurden durch die „Hilfspakete“ zum größten Teil von der EZB und dem ESM (Europäischer Stabilitätsmechanismus oder kurz „Rettungsschirm“) übernommen. Wenn ein griechischer Kreditausfall nicht die Profite der Banken schmälert, sondern aus dem Steueraufkommen bezahlt werden muss, dann haben wir das Merkel und Schäuble zu verdanken, die diese Art der Bankenrettung in den Jahren ab 2010 forciert haben.

Dass die Politik von Merkel und Schäuble für den deutschen Staat noch teuer werden wird, ist ziemlich sicher. Denn entweder kommt der Schuldenschnitt doch noch oder zumindest ein Teil der Bürgschaften, die bis jetzt einen Umfang von über 80 Milliarden Euro erreicht haben, wird fällig werden. Soweit, aber auch nur soweit, haben der deutsche Stammtisch und seine politischen Repräsentanten recht. Trotzdem machen Merkel und Schäuble gute deutsche Politik, sowohl in ökonomischer als auch in politischer Hinsicht.

Ökonomisch sind der deutsche Staat und das deutsche Kapital die riesigen Gewinner der Eurokrise. Angesichts des schwachen Euro floriert die deutsche Exportwirtschaft wie nie und die Arbeitslosenquote ist so niedrig wie seit Anfang der 1990er Jahre nicht mehr. Der deutsche Staat hat stark wachsende Steuereinnahmen - und für seine Schulden muss er kaum noch Zinsen zahlen, denn deutsche Staatsanleihen gelten als sicher und sind gesucht. Als Zugabe gibt es noch einen Braindrain aus den Krisenländern: Es kommen jede Menge gut ausgebildeter junger Menschen, die bereit sind, für wenig Geld zu arbeiten, da fallen die Defizite des deutschen Bildungssystems nicht mehr ganz so stark ins Gewicht. Aber beschränken wir uns auf die Zinsersparnis: Sie liegt nach jüngst veröffentlichten Berechnungen des Leibniz Instituts für Wirtschaftsforschung in Halle seit 2010 bei insgesamt ca. 100 Milliarden Euro und ist damit bereits höher als der Gesamtbetrag der deutschen Bürgschaften. Und diese Zinsersparnis hört ja nicht auf. Selbst wenn der deutsche Staat ab morgen wieder "normale“, d.h. nur wenig vom europäischen Durchschnitt abweichende Zinsen zahlen müsste, wie dies vor der Krise der Fall war, würden die niedrig verzinsten Papiere, die in den letzten Jahren ausgegeben wurden, weiterlaufen. Dass sich die Zinsen morgen normalisieren, ist aber nicht zu erwarten. Man kann durchaus prognostizieren, dass der deutsche Staat in den nächsten fünf Jahren noch mal die gleiche Summe an Zinskosten einspart, wahrscheinlich sogar erheblich mehr. Die Bürgschaftsmilliarden, die irgendwann mal fällig werden, lassen sich leicht verschmerzen, sie sind 
nur ein Bruchteil der bereits eingefahrenen Krisengewinne.

Politisch können sich die Resultate der Politik von Merkel und Schäuble ebenfalls sehen lassen. Die Eurozone wurde auf die deutsche Austeritätspolitik, zu der schon die rot-grüne Koalition einen kräftigen Beitrag geleistet hatte, eingeschworen. Während der Krise, das sei nur am Rande bemerkt, wurde diese Politik in Deutschland nicht angewandt, da gab es die Abwrackprämie und eine Verlängerung des Kurzarbeitergeldes. Und eine breite Zustimmung zu Merkel, was für das harte Auftreten nach außen nicht unwichtig ist. Die Eurozone aber wird so getrimmt, wie es sich die deutsche Regierung wünscht. Und eine linke Regierung, die, wie das Referendum vom 5. Juli 2015 zeigte, auch eine breite Mehrheit der Bevölkerung hinter sich versammeln konnte, wird in Rekordzeit in die Knie gezwungen. Normalerweise dauert es 15 oder 20 Jahre, bis eine kleine, mehr oder weniger radikale Partei an die Regierung kommt - als Juniorpartner in einer Koalition. Zeit genug ihr die radikalen Flausen auszutreiben, siehe z.B. die deutschen Grünen (auch wenn sich deren Radikalität immer schon in Grenzen hielt). In Krisenzeiten kann das alles jedoch viel schneller gehen: Syriza, 2009 noch eine linke Kleinpartei mit 4,6 Prozent der Stimmen, führt sechs Jahre später die Regierung. So schnell sollte das im demokratischen Europa eigentlich nicht gehen. Da sollte nur an die Regierung kommen, wer auch „verantwortungsbewusst“ handelt. Indem die deutsche Regierung Syriza in die Knie zwang, wurde auch dem Rest der EU klar gemacht: Es mag zwar Alternativen geben und ihr könnt sie auch wählen, aber sie werden keine Chance haben.

Keynesianisch orientierte Ökonomen und sozial orientierte Zeitgenossen laufen Sturm gegen diese Politik, sie befürchten, dass nicht nur die griechische Ökonomie, sondern auch das deutsche Ansehen und damit die deutsche Position in Europa und der Welt ganz fürchterlich beschädigt werden. Doch diese Politik entspringt keineswegs dem Starrsinn Schäubles oder seiner Unkenntnis elementarer ökonomischer Zusammenhänge. Diese Politik hat ihre eigene - machtpolitische - Logik. Mit dem Zusammenbruch der Sowjetunion verschwand der Ost-West Konflikt, mit dem all die vielen anderen Konflikte zugedeckt wurden. Die USA blieben zwar als einzige Supermacht übrig, doch ihre weltpolitische Dominanz hat abgenommen. Mit den Kriegen in Afghanistan und Irak wurde deutlich, dass die USA zwar jede Armee der Welt vernichtend schlagen können, der militärische Erfolg aber keineswegs bedeutet, sie könnten die Nachkriegsordnung uneingeschränkt bestimmen. Im ersten Jahrzehnt des neuen Jahrtausends schlug die Stunde der Mittelmächte. Brasilien und ganz Lateinamerika betreiben eine Politik, die unabhängiger von den USA ist, als dies seit Jahrzehnten der Fall war. China ist zum weltweiten Global Player geworden, sowohl auf politischer wie auf ökonomischer Ebene. Russland konnte zwar nicht an den Supermachtstatus der Sowjetunion anknüpfen, ist als Mittelmacht aber nicht zu unterschätzen. Und in Westeuropa? Da ist Deutschland schon längst die politisch und ökonomisch führende Macht, die zunehmend weltpolitische Ambitionen entwickelt. Nicht die weltweiten Militäreinsätze, an denen sich Deutschland inzwischen beteiligt, sondern der Euro und die Kontrolle der Eurozone sind dafür der Ausgangspunkt. Für große Teile des deutschen, wie auch der exportorientierten Teile des europäischen Kapitals, ist die Eurozone längst nicht mehr groß genug. Sie ist lediglich die Basis, von der die weltumspannenden Exportoffensiven ausgehen. Dafür muss die Eurozone aber 
auch entsprechend dem deutschen Modell ausgerichtet werden. Dazu gehört nicht nur eine stabile Währung, sondern auch den Sozialstaat auf eine Minimalsicherung zu beschränken und Schutzrechte der Beschäftigten abzubauen. Der weltweite Wettbewerb ist hart, da dürfen nicht schon „zuhause“ die Profite des Kapitals eingeschränkt werden. Genau das ist der Sinn der von Merkel und Schäuble forcierten Politik. Und nichts anderes ist gemeint, wenn als Ziel ausgegeben wird, aus der EU den „wettbewerbsfähigsten Wirtschaftsraum der Welt" zu machen. Dass Merkel und Schäuble ihr Programm durchsetzen können, liegt nicht allein an der deutschen Übermacht in der EU oder der Brutalität, mit der sie vorgehen. Das von ihnen verfolgte Programm trifft sich durchaus mit den Interessen jener Kapitalfraktionen der anderen EU-Länder, die fit für den Weltmarkt sind und sich der aggressiven deutschen Exportstrategie anschließen wollen. Was der deutschen Politik in den letzten Jahrzehnten innerhalb der EU gelungen ist, mit Lohnzurückhaltung und Austerität dem deutschen Exportkapital Vorteile zu verschaffen, soll jetzt auf der nächsthöheren Stufe organisiert werden. Mit der von der deutschen Regierung dominierten Eurozone wird eine starke Bastion für diesen globalen Konkurrenzkampf errichtet.

$\mathrm{Ob}$ diese sowohl nach innen (Austerität) als auch nach außen (Exportoffensive) äußerst aggressive Politik langfristig Erfolg haben wird, lässt sich nicht vorhersagen. Auf jeden Fall wird sie auf Weltebene mit weit stärkeren Gegnern konfrontiert sein, als sie es auf der europäischen Ebene jemals war. Allerdings zeigen sich die Bruchstellen dieser von der deutschen Regierung forcierten Politik auch auf der EU-Ebene. Zum einen sind es die zunehmenden nationalistischen Bewegungen in einzelnen Ländern der EU, die durch die Politik des deutschen Diktats Auftrieb erhalten. Zum anderen ist es Großbritannien, das deutlich andere weltpolitische und ökonomische Interessen hat als die übrigen EU-Mitgliedsländer. Politisch ist Großbritannien viel stärker mit den USA verbunden als jedes andere europäische Land, was sich nicht zuletzt darin zeigt, dass es zur Spionageallianz der "Five Eyes“ (USA, UK, Kanada, Australien und Neuseeland) gehört, die nicht gegeneinander spionieren, sondern miteinander den Rest der Welt ausforschen. Ökonomisch ist es nicht die britische Industrie, sondern vor allem der Finanzplatz London, der profitabel und politisch einflussreich ist und sich nicht der EU-Kontrolle oder gar dem Regime des Euro unterwerfen will. Insofern ist es nicht überraschend, dass die deutsche Politik das Lager der britischen EU-Gegner stärkt.

Bei guter deutscher Politik mag auch die SPD nicht abseitsstehen. Wer in dieser Koalition Koch und wer Kellner ist, versucht Sigmar Gabriel durch besonders lautes Schreien vergessen zu machen. Anlässlich des griechischen Referendums inszenierte er sich via Bild-Zeitung als besonders großer Griechenfresser. Häufig ist der Mann aber nur peinlich. Dies wurde deutlich, als Schäuble den "Grexit aufZeit" aus dem Hut zauberte und damit deutlich machte, dass er, um die griechische Regierung zur Unterwerfung zu zwingen, auch zu einer riskanten Politik bereit sei - niemand kann die Folgen eines Grexit nicht nur für Griechenland, sondern auch für den Euro wirklich abschätzen. Auf Nachfragen von Journalisten erklärte Gabriel zuerst vollmundig, selbstverständlich sei das mit ihm abgesprochen. Als der Unmut in der SPD überraschend schnell wuchs, schob er nach, dass es sich nur um eine Idee gehandelt habe, von der er mal gehört hätte.

Die veränderte Situation, in der deutsche Politik heute stattfindet, wird erst richtig deutlich, wenn man über die Europapolitik hinausschaut. Dazu nur zwei Beispiele. Vier 
Wochen lang streikten im Juni und Juli die Postangestellten: nicht in erster Linie für höhere Löhne, sondern für die Auflösung von neu gegründeten Tochtergesellschaften, in denen für die gleiche Arbeit bis zu 20 Prozent weniger Lohn gezahlt wird. Begründet wurde diese Lohnsenkung mit dem Erhalt der "Wettbewerbsfähigkeit“. Dabei hat der Konzern einen Vorsteuergewinn von über 3 Milliarden Euro und ist Marktführer. Der Streik endete, zumindest was die Hauptforderung nach Auflösung der neu gegründeten Gesellschaften angeht, mit einer völligen Niederlage. Auch in Deutschland wird das Kapital deutlich aggressiver und es hat Erfolg dabei.

Und zweitens TTIP, das Freihandelsabkommen zwischen der EU und den USA. Der Abbau von Zöllen ist dabei das geringste Problem. Wichtiger sind die Angleichungen von Umweltschutzund Verbraucherstandards, da wird es mit ziemlicher Sicherheit sowohl in den USA als auch in der EU deutlich nach unten gehen. Aber vor allem die Schiedsgerichte: Ursprünglich einmal eingeführt, um ausländische Investoren gegen entschädigungslose Enteignung in Staaten mit zweifelhaftem Rechtssystem zu schützen, geht es inzwischen nicht mehr nur um getätigte Investitionen, sondern um erwartete Profite, die durch staatliches Handeln eingeschränkt werden. So wie Griechenland jetzt erst einmal alle wichtigen Gesetzentwürfe in Brüssel vorlegen muss, bevor sie überhaupt dem Parlament zugeleitet werden, wird in Zukunft bei wichtigen Gesetzesvorhaben immer mit einberechnet werden müssen, wie hoch die Entschädigungsforderungen internationaler Konzerne sein könnten. Bei TISA, dem Abkommen über den Handel mit Dienstleistungen, das gerade verhandelt wird, und CETA, dem Freihandelsabkommen der EU mit Kanada, das schon weitgehend ausverhandelt ist, geht es noch um weitergehende Punkte, wie etwa das Verbot, privatisierte Unternehmen jemals wieder in die öffentliche Hand zu überführen. Bye, bye Rekommunalisierung. Mit TTIP, CETA und TISA würde die Position des Kapitals gegenüber jeder zukünftigen Regierung, egal, von wem diese gestellt wird, enorm gestärkt - auch wieder kein Konstruktionsfehler, sondern genau so gewollt. Aber das ist nicht alles. Nachdem die Liberalisierung des Welthandels über die Welthandelsorganisation WTO, doch nicht so besonders schnell vorankommt, wurden regionale Freihandelsabkommen wie TTIP oder das pazifische Freihandelsabkommen TPP (Trans-Pacific Partnership), das die USA gerade mit Pazifikanrainern verhandeln, wichtiger. Bei allen diesen Abkommen geht es auch darum, Standards zu formulieren, hinter die dann auch Abkommen nicht mehr zurückgehen können, die die USA und die EU zukünftig mit China, Indien und anderen Staaten schließen werden.

Gegen TTIP hat sich insbesondere in Deutschland ein breiter Widerstand formiert, der bis in weite Teile der SPD hineinreicht. Hier wird Sigmar Gabriel vielleicht seine historische Stunde erleben. Zunächst versuchte er, die Kritik an TTIP herunterzuspielen. Eine erste Unterschriftensammlung gegen TTIP kommentierte er mit dem Spruch, dass hier gegen etwas protestiert würde, dass es noch gar nicht gibt. Toller Gabriel-Witz. Wenn es TTIP erstmal gibt, nutzen auch Proteste nichts mehr. Als sich die Kritik innerhalb der SPD nicht mehr ignorieren ließ, äußerte Gabriel plötzlich Zweifel an ,privaten "Schiedsgerichten, was bei vielen SPD Mitgliedern für erleichtertes Aufatmen sorgte. Auch Gabriel aufder richtigen Seite. Mit den Handelsgerichten, die er ins Spiel brachte, ändert sich zwar nichts an der Sache, am Ende werden es aber genau solche kosmetischen Veränderungen sein, mit denen Gabriel gegenüber seiner SPD 
begründen wird, dass er trotz immer noch bestehender Bedenken für TTIP ist, da ein Scheitern noch schlimmer wäre. Und die Mitglieder und Abgeordneten der SPD, die vor nichts so viel Angst haben, wie gegen ihre Parteiführung aufzubegehren - so etwas schwächt die Partei - werden ihrem Vorsitzenden brav folgen.

Die Bevölkerung kann sich zwar die Regierung wählen, die sie haben möchte, der Spielraum dieser Regierung gegenüber dem Kapital wird aber immer weiter eingeschränkt. „Marktkonforme Demokratie" eben. Wie hieß es schon 2011 bei Merkel: Wir leben ja in einer Demokratie und deshalb „werden wir Wege finden, die parlamentarische Mitbestimmung so zu gestalten, dass sie auch marktkonform ist". Parlamentarische Mitbestimmung als notwendiges Übel, das man entsprechend zurechtstutzen muss, das ist kein Merkel'scher Ausrutscher. Das ist der Stand der Demokratie im globalen Konkurrenzkapitalismus. Die EU-Institutionen und insbesondere die Abgabe des nationalen geldpolitischen Einflusses durch die Einführung des Euro sind für diese "marktkonforme Demokratie“ auf europäischer Ebene wichtige Bausteine. Ein großer Teil der Linken hierzulande tut sich schwer mit einer grundsätzlichen EU-Kritik. Man will nicht in die rechte, nationalistische Ecke gestellt werden.

Aber das ist eine falsche Gegenüberstellung. Die EU und der Euro sind keine „eigentlich“ guten Einrichtungen, die nur an einem „Demokratiedefizit" leiden und unglücklicherweise von Politikern beherrscht werden, die immer noch auf eine destruktive "neoliberale" Politik setzen und die wunderschöne „europäische Idee“ von Völkerverständigung und Eierkuchen beschädigen. Bereits mit dem Maastricht-Vertrag, der "finanzielle Stabilität" (niedrige Inflationsraten und Schuldenbegrenzung) über alles stellt und sich über Steuer- und
Sozialsysteme ausschweigt, zwingt die Staaten in eine Situation, in der sie einerseits mit niedrigen Einkommens-, Gewinn- und Vermögenssteuern um Kapitalinvestitionen werben und andererseits aufgrund geringer Steuereinnahmen und „zu hoher" Verschuldung gar nicht anders können als Sozialleistungen zu kürzen. Das ist kein Mangel des Euro, das ist die Logik des Euro, die von den Regierungen, die ihn einführten, auch genauso gewollt war. Während das „Mehrebenensystem“ der EU den stärkeren Ländern zwar mehr Einfluss gibt, aber auch Blockademöglichkeiten der schwächeren Länder einschließt, hat sich die Lage mit der Einführung des Euro dramatisch geändert. Was sich schon im Fall Zypern andeutete, wurde am Beispiel Griechenlands unübersehbar: Ein schwaches, verschuldetes Land hat keine Chance, wenn die stärksten Mächte (oder auch nur die stärkste Macht) im Verbund mit der EZB agieren, Mehrheiten hin oder her. Und weil das jetzt auch alle wissen, werden die anderen Länder alles daran setzen, nicht in eine Situation zu kommen wie Griechenland.

Die Annahme eines großen Teils der Linken hierzulande, dass es doch besser sei, im Innern der EU für progressive Veränderungen zu kämpfen als außerhalb, gilt seit Einführung des Euro zumindest für kleine, wirtschaftlich schwächere Länder definitiv nicht. Außerhalb der Eurozone wären die Bedingungen für die SyrizaRegierung erheblich besser. Aber ein "Grexit" hätte für Griechenland zum jetzigen Zeitpunkt unkalkulierbare Folgen, das Rad der Geschichte lässt sich nicht ohne Weiteres zurückdrehen. Doch künftige Beitrittskandidaten sollten sich die Sache genau überlegen. Der Euro, der in vielen Ländern mit schwachen Währungen für Stärke, Stabilität, niedrige Zinsen und niedrige Inflationsraten steht, könnte sich für sie als ein vergiftetes Geschenk erweisen. 